
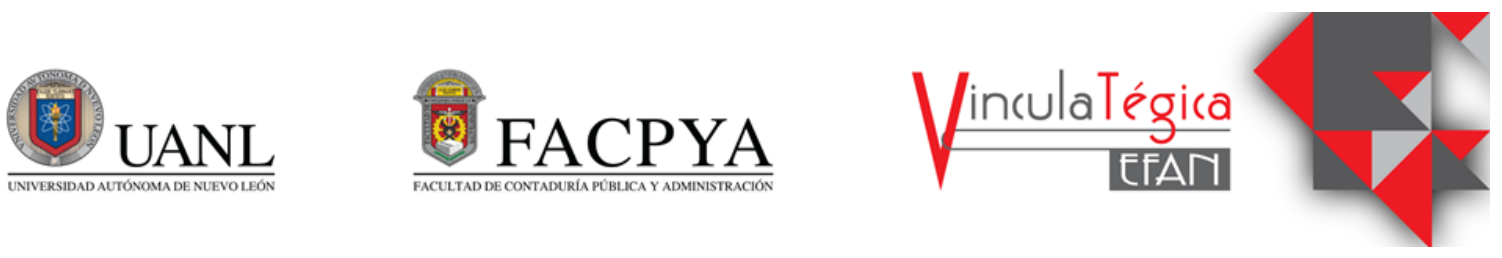

\title{
El comportamiento en los niveles de pobreza y política educativa como solución, un análisis para la Ciudad de México y Estado de México
}

\author{
Luis Antonio Andrade Rosas ${ }^{1}$, Alma Cosette Guadarrama Muñoz y Gaytán Alcalá Felipe ${ }^{3}$ \\ ${ }^{I}$ Profesor investigador de la Facultad de Negocios de la Universidad La Salle, Ciudad de México, \\ luis.andrade@lasalle.mx, Av.Benjamín Franklin 45, Col. Condesa, Alc. Cuauhtémoc, Ciudad de México. \\ +(52) 5552789500 , ext. 3112. \\ ${ }_{2}^{2}$ Investigadora de la Facultad de Derecho de la Universidad La Salle, Ciudad de México, \\ lancelot56@gmail.com, Av.Benjamín Franklin 45, Col. Condesa, Alc. Cuauhtémoc, Ciudad de México. \\ $+(52) 5552789500$, ext. 3112 . \\ ${ }^{3}$ Profesor investigador de la Facultad de Negocios de la Universidad La Salle, Ciudad de México, \\ felipe.gaytan@lasalle.mx, Av.Benjamín Franklin 45, Col. Condesa, Alc. Cuauhtémoc, Ciudad de México. \\ $+(52) 5552789500$, ext. 3112.
}

Información del artículo revisado por pares

Fecha de aceptación: junio-2021

Fecha de publicación en línea: diciembre-2021

DOI: https://doi.org/10.29105/vtga7.1-154

\section{Resumen}

La pobreza es un problema que afecta a toda la sociedad de un Estado, en particular a las familias. En este trabajo se analizan las condiciones de pobreza extrema de manera conjunta para la Ciudad de México y el Estado de México. El análisis es a través de tablas de frecuencias y expresiones lineales, en donde se muestra principalmente que conforme los porcentajes de pobreza van aumentando, hay pocos municipios que cumplen estas condiciones precarias de pobreza, adicional se muestra una relación positiva entre los rezagos educativos y los índices de pobreza extrema. Como resultado, y a partir de las condiciones precarias de pobreza, se muestra que la política educativa puede ser una posible solución para disminuir los índices de pobreza extrema. Si bien, el análisis sirve para evaluar la intervención gubernamental a través de la educación, faltaría averiguar si existen las condiciones para emplear a la mayoría de las personas que disminuyeron su rezago educativo.

Palabras clave: Pobreza, rezago educativo, gasto público.
Abstract

Poverty is a problem that affects the entire society of a State, particularly families. Extreme poverty conditions for the municipalities of Mexico City and the State of Mexico are analyzed. The analysis is through tables of frequencies and linear expressions, where it is mainly shown that as the percentages of poverty increase, there are few municipalities that meet these precarious poverty conditions. In addition, a positive relationship is shown between educational lags and extreme poverty rates; In this way, it can be deduced that educational policy can be a possible solution to reduce extreme poverty rates. Although the analysis serves to evaluate government intervention through education, it remains to be ascertained whether the conditions exist to employ the majority of the people who reduced their educational gap.

Keywords: Poverty, educational, backwardness, public spending.

JEL: I32, I22, H54. 


\section{INTRODUCCIÓN}

Las condiciones de pobreza que vive un país son cruciales para su desarrollo, al ser los principales afectados las familias. Existen varias formas de medir la pobreza, en este trabajo se retoma la pobreza extrema, que se define como aquella en donde la población vive con menos de 1.9 dólares al día (Banco Mundial, 2020). Afortunadamente, el Banco Mundial señala que estos niveles de pobreza extrema disminuyeron para 2017 aproximadamente en $1 \%$. Si bien el análisis retoma un índice de pobreza dado, hay formas para medir estos niveles de pobreza; por ejemplo, Ortiz y Bolívar (2013) proponen diferentes índices para medir la pobreza en México a mediados del 2010.

Existen varias estrategias para erradicar los niveles de pobreza, como el crecimiento, el empleo y el nivel de educación. No obstante, los esfuerzos en estas políticas no han llevado a los objetivos planteados, sino por el contrario, se han visto estancados en niveles de pobreza (Andrade y Jiménez, 2018). De esta forma, las familias tienen que optar por estrategias propias, como lo señalan Naude y Andrade (2008), a través de un análisis de efectos multiplicadores, comentan que la agricultura para las familias rurales fue determinante para aumentar las condiciones de ingresos de los pobladores y con ello disminuir los niveles de pobreza.

En este trabajo se analizan las condiciones de pobreza extrema para los 125 municipios del Estado de México y las 16 alcaldías de la Ciudad de México. El análisis, a través de tablas de frecuencia, muestra en particular que hay siete municipios en donde uno de cada cuatro pobladores vive con menos de 1.9 dólares al día, lo cual es preocupante. De esta forma, el analisis recomienda la intervención gubernamental, la cual se presenta a través de la diminución de rezago educativo que tiene efecto en la reducción de los niveles de pobreza extrema.

El trabajo se estructuró bajo el siguiente esquema, adicional a la introducción, se muestra el marco referencial de pobreza y sus posibles soluciones; posteriormente, se presenta la metodología de tablas de frecuencias y expresiones lineales. El apartado 4 corresponde al análisis y los resultados de las condiciones de pobreza y el nivel de educación como posible solución. Finalmente, se presenta una breve conclusión con la discusión pertinente respecto a los resultados obtenidos y la literatura consultada.

\section{MARCO TEÓRICO}

A lo largo de la historia se ha diseñado política social para erradicar problemas de pobreza que sufren los individuos, ya sea en zonas urbanas o rurales. Por ejemplo, Lorenzo (2018) habla de los estigmas y las normas que definieron la pobreza urbana para articular la política social hacia los mendigos de la Ciudad de México, alrededor de los años 30. Se han optado por diferentes estrategias para erradicar los niveles de pobreza, que van desde educación, empleo, apertura comercial, crecimiento económico, entre otros. Al respecto, Vázquez y Monroy (2016), comentan que el nivel de crecimiento es clave para disminuir los índices de pobreza alimentaria siempre que sea bien estructurada la estrategia, adicional comentan que, si bien el empleo originado por este crecimiento es clave para la disminución de la pobreza, el salario percibido en estos empleos aun no lo es.

A pesar de que la política para la disminuir los índices de pobreza es loable, hay que tener cuidado en la forma en que se distribuyen los recursos, las oportunidades, y los apoyos, entre otros. Porque una cosa es un gobierno loable y "gentil" y otra que estas estrategias se utilicen como arma política. En este sentido, MacEwan (2010) sustenta que cuando el poder se toma en cuenta, el objetivo de la política cambia hacia cuestiones como reformas agrarias, al tener como consecuencia que en lugar de obtener "cosas" para los pobres, la política pudiera crear condiciones para que los pobres adquieran poder.

En particular los países emergentes son los 
que viven en condiciones más precarias, y los niveles de pobreza se localizan sobre todo en comunidades rurales, que obligan a los ciudadanos a migrar a las metrópolis. No obstante, se ha observado que la migración en la búsqueda de trabajo no es la solución, sino que las autoridades deberían de comprometerse y preocuparse por las necesidades de quienes habitan en zonas rurales para disminuir sus condiciones de desigualdad y carencias sociales (Salama, 2011).

Por otra parte, los factores más importantes que causan las condiciones de pobreza son el nivel de ingreso y el desempleo. Por ejemplo, en esta época de pandemia se ha observado que las condiciones de salarios son ineficientes para alcanzar al menos una canasta básica. Estas condiciones de pobreza determinada por bajos salarios, Tejero (2017) la define como pobreza laboral.

\section{MÉTODO}

La metodología en principio es a través de un análisis estadístico y gráfico. El primer concepto requerido es un histograma cuya definición es,

Definición 1. Un histograma es un gráfico de barras, que acumula la información en orden creciente. Los componentes principales del histograma son,

i) El número de intervalos, también llamados clases,

ii) la anchura de cada intervalo,

iii) la altura de los intervalos.

El número de intervalos se encuentra de acuerdo a,

$$
\text { Núm de int } \approx \sqrt{n}
$$

donde $n$ es el tamaño muestral o el número de datos de la muestra. Por su parte, la anchura de cada intervalo se encuentra a partir de,

$$
\text { Anch }=\frac{\text { Max-Min }}{\text { Núm de int }}
$$

donde Max y Min se refieren al número más grande y más pequeño respectivamente de la información. Finalmente, la frecuencia hace referencia a la altura de los intervalos, es decir, cuanta información está en cada intervalo.

Un concepto importante en la construcción del histograma, llamado frecuencia que no es más que la probabilidad, que se puede definir a través de la siguiente definición,

Definición 2. Sea $X$ una variable aleatoria, entonces la frecuencia relativa se puede "atrapar" a través del concepto de probabilidad marginal de la siguiente forma,

$$
p(X) \approx P(a \leq X \leq b)
$$

donde $a$ y $b$ hace referencia al inicio y final de cualquier intervalo, o la característica que guarda el intervalo. Respecto a la frecuencia acumulada relativa, se tiene el siguiente concepto,

Definición 3. Sea $X$ una variable aleatoria, entonces la frecuencia acumulada relativa $F_{X}(x)$ se puede localizar mediante,

$$
F_{X}(a)=P(X \leq a)
$$

que se refiere a la probabilidad o frecuencia acumulada hasta el punto $a$.

Con base en los conceptos de probabilidad marginal y acumulada, se pueden detectar estadísticas que en general se llaman cuantiles, esto es,

Definición 4. Sea $X$ una variable aleatoria, entonces un cuantil $q$ hace referencia al hecho de que antes de este punto se tiene acumulado $q \%$ de probailidad, esto es,

$$
P\left(X \leq x_{q}\right)=q \%
$$

donde $x_{q}$ se refiere al valor donde se alcanza el $q \%$ de probabilidad. Por ejemplo, si se parte la información en 4, cada una de las partes se 
le llama cuartil. Esto es, si $P\left(X \leq x_{2}\right)=50 \%$, entonces el valor $x_{2}$ se le llama cuartil 2, decil 5 , centil 50 o simplemente mediana.

$\mathrm{Si}$ en datos agrupados no se puede detectar de forma precisa un decil o cuartil, o en particular la mediana, Canavos (1994) muestra cómo encontrar la mediana a partir de datos agrupados, cuya expresión es la siguiente,

$$
\text { mediana }=L+c(j / f r e c)
$$

donde,

$L$ es el inicio del intervalo donde está la mediana,

$c$ es la anchura del intervalo,

$j$ se refiere al número que hace falta para llegar a la mitad de la información desde el intervalo anterior a la mediana.

frec es la frecuencia relativa del intervalo donde está la mediana.

Finalmente, el análisis requiere una estrategia para combatir el analisis de pobreza, lo que se hace a través de una expresión lineal, que se define a continuación,

Definición 5. Una expresión lineal es una relación entre dos variables, una llamada dependiente $(Y)$ y otra llamada independiente $(X)$, la relación se expresa como,

$$
Y=a+b X
$$

donde $a$ se le conoce como intercepto $u$ ordenada al origen, representa el corte con el eje $Y$.

$b$ es la pendiente, y muestra el cambio que sufre $\mathrm{Y}$ debido a cambios en $X$, esto es,

$b=\frac{\Delta Y}{\Delta X}$, donde $\Delta Y$ es el cambio en $Y$ y $\Delta X$ es el cambio en $X$. Por ejemplo, i) si $b>0$ la relación entre $X$ y $Y$ es positiva y la recta va hacia arriba,

ii) si $b<0$, la relación entre $X$ y $Y$ es negativa y la recta va hacia abajo. para el caso i), si $X$ es el nivel de corrupción, y $Y$ es el nivel de pobreza, si hay mayor corrupción habrá mayores índices de pobreza, esto es, hay una relación positiva entre estas dos variables. Para el caso ii), imagine que $X$ es el nivel de educación y $Y$ es el nivel de pobreza, entonces a mayor educación, menor pobreza.

\section{RESULTADOS}

A partir de la base de datos sobre los niveles de pobreza que reporta Coneval (2015), se hará un análisis gráfico para observar la problemática de estos niveles de pobreza. En particular Coneval menciona tres tipos de pobreza para todos los municipios de México. En este trabajo, nos enfocaremos a analizar el porcentaje de personas en pobreza extrema, que de acuerdo al Banco Mundial (2020) son personas que viven con menos de 1.9 dólares al día. En particular para la zona conurbada de la Ciudad de México y del Estado de México.

\subsection{Comportamiento de la pobreza}

Suponga que se tienen los niveles de pobreza extrema y los niveles de rezago educativo, para los dos estados, Ciudad de México y Estado de México.

Sea $X_{P E x t r}$ el porcentaje de personas viviendo con menos de 1.9 dólares al día en los 141 municipios de la zona conurbada del valle de México, cuya distribución por clases se observa en la tabla 1. 
Tabla 1. Distribución de la pobreza extrema de la zona conurbada de la Ciudad de México.

\begin{tabular}{|r|r|r|r|r|r|r|r|r|}
\hline Intervalos & inicio & final & Frec Abs & Frec Relat & Fre Acumu Abs & FAR(frec Acum Relat) & marca: punto medio & PM*frecRel \\
\hline 1 & 0.1 & 2.89166667 & 24 & 0.17021277 & 24 & 0.170212766 & 1.495833334 & 0.25460993 \\
\hline 2 & 2.89166667 & 5.68333333 & 40 & 0.28368794 & 64 & 0.453900709 & 4.2875 & 1.21631206 \\
\hline 3 & 5.68333333 & 8.475 & 21 & 0.14893617 & 85 & 0.602836879 & 7.079166667 & 1.05434397 \\
\hline 4 & 8.475 & 11.2666667 & 18 & 0.12765957 & 103 & 0.730496454 & 9.870833335 & 1.26010638 \\
\hline 5 & 11.2666667 & 14.0583333 & 9 & 0.06382979 & 112 & 0.794326241 & 12.6625 & 0.80824468 \\
\hline 6 & 14.0583333 & 16.85 & 4 & 0.02836879 & 116 & 0.822695035 & 15.45416667 & 0.43841608 \\
\hline 7 & 16.85 & 19.6416667 & 6 & 0.04255319 & 122 & 0.865248227 & 18.24583334 & 0.77641844 \\
\hline 8 & 19.6416667 & 22.4333333 & 6 & 0.04255319 & 128 & 0.907801418 & 21.0375 & 0.89521277 \\
\hline 9 & 22.4333333 & 25.225 & 6 & 0.04255319 & 134 & 0.95035461 & 23.82916667 & 1.01400709 \\
\hline 10 & 25.225 & 28.0166667 & 1 & 0.0070922 & 135 & 0.957446809 & 26.62083334 & 0.18880024 \\
\hline 11 & 28.0166667 & 30.8083333 & 3 & 0.0212766 & 138 & 0.978723404 & 29.4125 & 0.62579787 \\
\hline 12 & 30.8083333 & 33.6 & 3 & 0.0212766 & 141 & 1 & 32.20416667 & 0.68519504 \\
\hline
\end{tabular}

Fuente: Elaboración propia con datos de Coneval (2015).

Dentro de los resultados de la tabla 1 , note en la última fila de la frecuencia absoluta que hay 3 municipios que rebasan el $30 \%$ en que su población está en pobreza extrema. Con base en la información de los municipios que se presenta en el anexo, se puede observar que tales municipios son: Donato Guerra, Luvianos e Ixtapan del Oro. Se observa también, que el porcentaje de población viviendo en condiciones de pobreza extrema la mayoría ocurre entre $2.89 \%$ y $5.60 \%$. En estos porcentajes de pobreza hay 40 municipios, que corresponden al $28 \%$ de la información. Finalmente, sobresale el hecho que las 16 alcaldías de la Ciudad de México son las menos pobres, es decir, el porcentaje de población en pobreza extrema no rebasa el $2.89 \%$, lo que puede verificarse al observar la información de los municipios en la tabla 2 del anexo.

El histograma de la figura 1 muestra la distribución de estos niveles de pobreza, en donde se aprecia una caída de los porcentajes de población en pobreza que van aumentando, es decir, hay menos personas o municipios viviendo en condiciones de pobreza extrema.

En la misma figura se muestra un sesgo de la información a la derecha, una de las características de este sesgo es que la media es mayor a la mediana. Como se citó en la metodología se pueden encontrar estos valores para datos agrupados. Para obtener la media con datos agrupados, se utilizan las últimas dos columnas de la tabla 1 , siendo en este caso la media igual a 9.2117, valor que cae en el cuarto intervalo. Así, a partir de la FAR (frecuencia acumulada relativa) se observa que por debajo de este promedio hay aproximadamente 103 municipios equivalente al $73 \%$ del total, esto es, el valor de la media no corresponde con la mediana, el valor de la mediana es más bajo.

La mediana y otros percentiles se pueden encontrar a partir de los conceptos probabilísticos vistos en la metodología. En particular,

i) En el intervalo 8 se observa que, $P(X \leq 22.433)=0.90$, de esta forma el valor 22.433 se le llama centil 90, o decil 9.

ii) análogamente, se observa en el intervalo 3 que hasta el punto 8.475 se acumula el $60 \%$ de la población. Y de esta forma, el valor 8.475 se denomina centil 60 o decil 6.

No obstante, en la tabla 1 no se puede observar la mediana, sabiendo que se tiene que cumplir lo siguiente,

$P(X \leq$ mediana $)=50 \%$, 
Figura 1. Histograma del nivel de pobreza extrema.

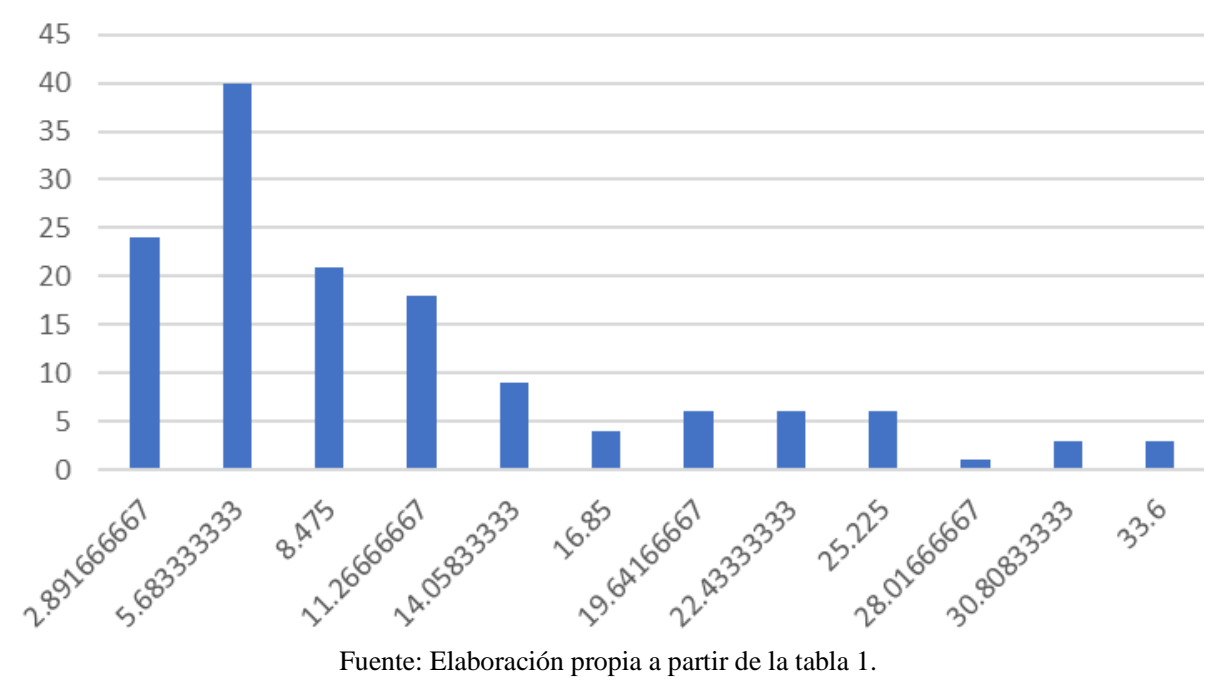

Con base en la expresión (5) y los valores encontrados se tiene que la mediana es, 6.5474. Esto es, $P(X \leq 6.547)=50 \%$, o simplemente, en el $50 \%$ de los municipios de El Estado de México y Ciudad de México, el $6.5474 \%$ de la población vive en extrema pobreza.

mostrar una estrategia como solución para disminuirla. En particular se propone disminuir los índices de rezago educativo y ver su efecto en el nivel de pobreza, esto es, se puede estimar la relación entre pobreza extrema $(Y)$ y el nivel de rezago educativo $(X)$, de esta forma, con la información del anexo para los dos Estados, se tiene que la relación es,

$$
Y=-5.0574+0.8488 X
$$

Dado el comportamiento de los niveles de pobreza en la sección anterior, es importante

El comportamiento de esta expresión se observa en la figura 2 .

Figura 2. Relación entre los niveles de pobreza y el rezago educativo. $\mathrm{Y}=\mathrm{f}(\mathrm{X})=-5.057+0.848 \mathrm{X}$

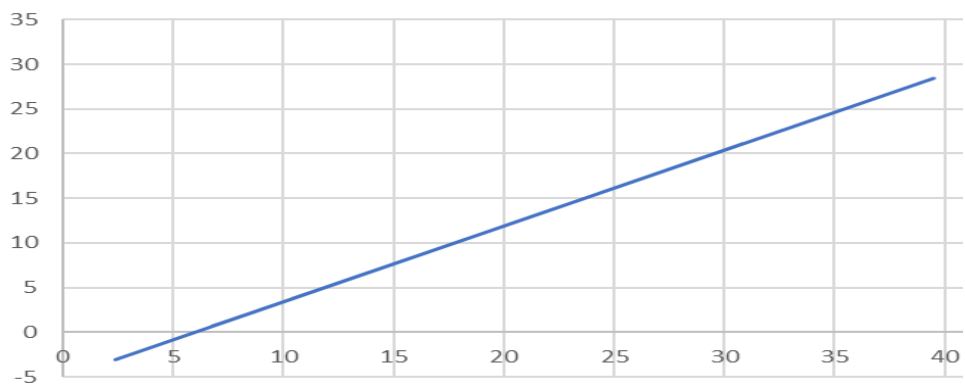

Fuente: Elaboración propia, con datos de Coneval (2015)

La expresión (6), al igual que la figura 2 , muestra que conforme haya más rezago educativo los niveles de pobreza aumentan. A partir de esto, se puede evaluar la estrategia del gobierno para combatir del rezago educativo. Por ejemplo, suponga que el gobierno invierte en educación y su efecto se traslada en una reducción del $15 \%$ en el rezago educativo, esto es, $\Delta X=-15<0$. Con base en la pendiente de la expresión (6), se tiene que,

$\frac{\Delta Y}{\Delta X}=0.848$, despejando y sustituyendo el cambio en el rezago educativo,

$$
\begin{gathered}
\Delta Y=0.848 * \Delta X=0.848 *(-15) \\
=-12.72
\end{gathered}
$$


lo que implica que la inversión en el rezago educativo para alcanzar una disminución del $15 \%$, tiene un efecto en la reducción de los niveles de pobreza de $12.72 \%$, lo cual es una cifra idónea, en el sentido que las personas podrían dejar situaciones críticas de pobreza extrema.

Adicional, se puede relacionar la expresión (6) con la tabla 1 de frecuencias para alcanzar objetivos específicos. Por ejemplo, suponga que el gobierno tiene como objetivo no rebasar el sexto decil de los niveles de pobreza extrema, esto es, que el porcentaje de la población en extrema pobreza no sea mayor a $8.475 \%$, entonces, el nivel de rezago educativo que habría que considerar para no rebasar este nivel de pobreza precaria, se encuentra a partir de,

$Y=-5.0574+0.848 X \leq 8.475$

$0.848 X \leq 13.5324$, finalmente, $X \leq 15.95$.

Así, si las autoridades quieren evitar que el sexto decil de la población no rebase el hecho de que 8 de cada 100 personas vivan en pobreza extrema, deben invertir en infraestructura correspondiente en educación a fin de que la población no rebase el 16 por ciento de rezago educativo.

\section{CONCLUSIONES}

En este trabajo se analizó el comportamiento, a través de una tabla de frecuencias, de los niveles de pobreza extrema para la Ciudad de
México y el Estado de México de manera conjunta. Los resultados muestran que las alcaldías de la Ciudad de México son las que tienen menor porcentaje de población viviendo en extrema pobreza, en comparación con el Estado de México, en donde la situación es a la inversa. En particular, se observan que, en siete municipios, uno de cada cuatro pobladores vive en extrema pobreza.

La educación puede ser una herramienta para reducir los índices de pobreza de cualquier población, al respecto Salvador (2008) muestra como la educación en México puede alentar a las personas para un amplio desarrollo y con ello salir de los niveles de pobreza. Al respecto, Navarro, Lenin y Ortega (2017), con base en un análisis de manera general para México en 2014, muestran como el uso óptimo de los insumos como el producto interno bruto y el gasto en educación, hacen que el nivel de educación superior sea un factor clave para erradicar los problemas de pobreza.

Si bien el nivel de educación es factor para salir de las condiciones precarias de pobreza y fomentar un desarrollo optimo, faltaría ver si la gente que sale del rezago educativo, realmente se emplea o puede acceder a una mejor calidad de vida. Es decir, además del rezago educativo que se va a combatir, faltaría involucrar variables como desempleo, ingresos, inversión, entre otras. Análisis que se dejan para futuras investigaciones.

\section{REFERENCIAS}

Andrade, L., y Jiménez-Bandala, C. (2018). El desempleo y la probabilidad de caer en trampas de pobreza: Consideraciones para países en vías de desarrollo. Reis: Revista Española De Investigaciones Sociológicas, (164), 3-20. DOI:10.2307/26633315

Banco Mundial (2020). Pobreza: Panorama general. Pobreza: Panorama general (bancomundial.org). (3 de febrero 2021)

Canavos, G. (1988). Probabilidad y estadística. Aplicaciones y métodos. Virginia: CommonWealth the University.

Coneval (2015). Indicadores de pobreza municipal, 2015. Datos Abiertos de México - Indicadores de pobreza municipal 2010 - 2015 - indicadores de pobreza municipal, 2015. (27 de enero 2021)

Lorenzo, M. (2018). El análisis de la pobreza y la campaña contra la mendicidad en la Ciudad de México, 1929-1931. Historia Mexicana, 67(4 (268)), 1677-1724.

MacEwan, A. (2010). El significado de la pobreza: Cuestiones de distribución y poder. Investigación 
Económica, 69(272), 15-56.

Naude, A., y Andrade, S. (2008). Efectos de los multiplicadores de las actividades productivas en el ingreso y pobreza rural en México. El Trimestre Económico, 75(298(2)), 349-377.

Navarro, C., Lenin, J., y Ortega, O. (2017). Educación y pobreza en México. Un análisis de eficiencia a nivel estados. Acta Universitaria, 27(1), 33-45.

Ortiz, J. y Ríos H. (2013). La Pobreza en México, un análisis con enfoque multidimensional. Análisis Económico, 28(69),189-218.

Salama, P. (2011). Luchas contra la pobreza en América Latina. El caso de la pobreza rural en Brasil. Problemas Del Desarrollo, 42(165), 7-34.

Salvador, L. (2008). Desarrollo, educación y pobreza en México. Papeles de población, (55), 237-257.

Tejero, A. (2017). Permanencia en la pobreza laboral: La influencia de la pobreza pasada en la presente. Reis: Revista Española De Investigaciones Sociológicas, (157), 141-161.

Vázquez, R., y Monroy-Gómez-Franco, L. (2016). La relación entre crecimiento económico y pobreza en México. Investigación Económica, 75(298), 77-113.

Anexo

\begin{tabular}{|c|c|c|}
\hline Entidad & Municipio & $\begin{array}{l}\text { Porcentaje de la población en pobreza } \\
\text { extrema }\end{array}$ \\
\hline Distrito Federal & Benito Juárez & \\
\hline Distrito Federal & Miguel Hidalgo & \\
\hline Distrito Federal & Iztacalco & \\
\hline Distrito Federal & Azcapotzalco & \\
\hline Distrito Federal & Coyoacán & \\
\hline Distrito Federal & Cuauhtémoc & \\
\hline Distrito Federal & Venustiano Carranza & \\
\hline Distrito Federal & Gustavo A. Madero & \\
\hline Distrito Federal & Álvaro Obregón & \\
\hline Distrito Federal & Cuajimalpa de Morelos & \\
\hline Distrito Federal & La Magdalena Contreras & \\
\hline Distrito Federal & Tláhuac & \\
\hline México & Cuautitlán & \\
\hline Distrito Federal & Iztapalapa & \\
\hline Distrito Federal & Tlalpan & \\
\hline Distrito Federal & Milpa Alta & \\
\hline México & Coacalco de Berriozábal & \\
\hline México & Chapultepec & \\
\hline México & Atizapán de Zaragoza & \\
\hline México & Huixquilucan & \\
\hline Distrito Federal & Xochimilco & \\
\hline México & Rayón & \\
\hline México & Huehuetoca & \\
\hline México & $\begin{array}{l}\text { Cuautitlán Izcalli } \\
\text { San Martín de las }\end{array}$ & \\
\hline México & Pirámides & \\
\hline México & San Antonio la Isla & \\
\hline México & Temamatla & \\
\hline
\end{tabular}




\begin{tabular}{|c|c|c|}
\hline México & Jilotzingo & 3.2 \\
\hline México & Metepec & 3.2 \\
\hline México & Polotitlán & 3.2 \\
\hline México & Tecámac & 3.2 \\
\hline México & Tlalnepantla de Baz & 3.2 \\
\hline México & Nopaltepec & 3.3 \\
\hline México & Tultitlán & 3.3 \\
\hline México & Jaltenco & 3.4 \\
\hline México & Tonatico & 3.4 \\
\hline México & Mexicaltzingo & 3.5 \\
\hline México & Nezahualcóyotl & 3.5 \\
\hline México & Cocotitlán & 3.7 \\
\hline México & Teotihuacán & 3.7 \\
\hline México & Tepotzotlán & 3.8 \\
\hline México & Lerma & 3.9 \\
\hline México & Naucalpan de Juárez & 3.9 \\
\hline México & Capulhuac & \\
\hline México & Ocoyoacac & \\
\hline México & Otumba & \\
\hline México & Papalotla & 4.1 \\
\hline México & Tenango del Aire & 4.1 \\
\hline México & Apaxco & 4.2 \\
\hline México & San Mateo Atenco & 4.3 \\
\hline México & Tultepec & 4.4 \\
\hline México & Tepetlaoxtoc & 4.5 \\
\hline México & Melchor Ocampo & 4.6 \\
\hline México & Zumpango & 4.6 \\
\hline México & Ayapango & 4.7 \\
\hline México & Nicolás Romero & 4.7 \\
\hline México & Axapusco & 4.8 \\
\hline México & Calimaya & 4.8 \\
\hline México & Ecatepec de Morelos & 4.9 \\
\hline México & Coyotepec & 5 \\
\hline México & Isidro Fabela & 5 \\
\hline México & Chiautla & 5.1 \\
\hline México & Tonanitla & 5.1 \\
\hline México & Chicoloapan & 5.3 \\
\hline México & Temascalapa & 5.7 \\
\hline México & Timilpan & 5.7 \\
\hline México & Xonacatlán & 5.7 \\
\hline México & Tlalmanalco & 5.9 \\
\hline México & Toluca & 6.1 \\
\hline México & Teoloyucan & 6.4 \\
\hline México & Tequixquiac & 6.4 \\
\hline México & Texcoco & 6.4 \\
\hline
\end{tabular}


México Soyaniquilpan de Juárez $\quad 6.6$

$\begin{array}{lll}\text { México } & \text { Nextlalpan } & 6.7\end{array}$

$\begin{array}{lll}\text { México } & \text { Atenco } & 6.8\end{array}$

$\begin{array}{lll}\text { México } & \text { Atizapán } & 6.9\end{array}$

$\begin{array}{lll}\text { México } & \text { Almoloya del Río } & 7.3\end{array}$

$\begin{array}{lll}\text { México } & \text { Amecameca } & 7.6\end{array}$

$\begin{array}{lll}\text { México } & \text { Zinacantepec } & 7.8\end{array}$

México $\quad$ Valle de Bravo $\quad 8$

México $\quad$ Hueypoxtla $\quad 8.1$

$\begin{array}{lll}\text { México } & \text { Ixtapaluca } & 8.1\end{array}$

$\begin{array}{lll}\text { México Jocotitlán } & 8.2\end{array}$

México $\quad$ Chiconcuac $\quad 8.3$

$\begin{array}{lll}\text { México } & \text { Texcalyacac } & 8.3\end{array}$

$\begin{array}{lll}\text { México } & \text { Acolman } & 8.6\end{array}$

México $\quad$ Tianguistenco $\quad 8.6$

$\begin{array}{lll}\text { México } & \text { Chalco } & 8.7\end{array}$

$\begin{array}{lll}\text { México } & \text { Ixtapan de la Sal } & 8.7\end{array}$

$\begin{array}{lll}\text { México } & \text { Xalatlaco } & 8.9\end{array}$

México $\quad$ Juchitepec $\quad 9.1$

México $\quad$ Chimalhuacán $\quad 9.2$

México $\quad$ Tenancingo $\quad 9.4$

México

Valle de Chalco

Solidaridad $\quad 9.5$

México

La Paz

9.6

Jilotepec $\quad 9.8$

México

Atlacomulco 10.4

$\begin{array}{lll}\text { México } & \text { Tenango del Valle } & 10.4\end{array}$

$\begin{array}{lll}\text { México } & \text { Aculco } & 10.7\end{array}$

$\begin{array}{lll}\text { México } & \text { Jiquipilco } & 10.7\end{array}$

$\begin{array}{lll}\text { México } & 10.7\end{array}$

México Ozumba 11

$\begin{array}{lll}\text { México } & \text { El Oro } & 11.1\end{array}$

México $\quad$ Otzolotepec 11.4

México $\quad$ Tepetlixpa 12

México $\quad$ Villa Guerrero 12.1

México Zacazonapan $\quad 12.2$

$\begin{array}{llr}\text { México } & \text { Coatepec Harinas } & 12.4\end{array}$

$\begin{array}{lll}\text { México } & \text { Almoloya de Juárez } & 12.6\end{array}$

$\begin{array}{lll}\text { México } & \text { Santo Tomás } & 12.7\end{array}$

México Ixtlahuaca 13

$\begin{array}{lll}\text { México } & \text { Chapa de Mota } & 13.8\end{array}$

$\begin{array}{lll}\text { México } & \text { Tezoyuca } & 14.8\end{array}$

México $\quad$ San Simón de Guerrero $\quad 15.2$

México Ocuilan 15.3

$\begin{array}{lll}\text { México } & \text { Atlautla } & 15.9\end{array}$ 
$\begin{array}{llr}\text { México } & \text { Amanalco } & 17.4 \\ \text { México } & \text { Villa del Carbón } & 17.5 \\ \text { México } & \text { Texcaltitlán } & 18.4 \\ \text { México } & \text { Almoloya de Alquisiras } & 18.7 \\ \text { México } & \text { Temoaya } & 18.7 \\ \text { México } & \text { Malinalco } & 19.4 \\ \text { México } & \text { Acambay } & 19.8 \\ \text { México } & \text { Temascalcingo } & 20.1 \\ \text { México } & \text { Ecatzingo } & 21.2 \\ \text { México } & \text { Villa Victoria } & 21.5 \\ \text { México } & \text { Tejupilco } & 21.6 \\ \text { México } & \text { Temascaltepec } & 22.1 \\ \text { México } & \text { Zumpahuacán } & 22.8 \\ \text { México } & \text { San José del Rincón } & 22.8 \\ \text { México } & \text { Morelos } & 23.8 \\ \text { México } & \text { Amatepec } & 23.9 \\ \text { México } & \text { San Felipe del Progreso } & 24.5 \\ \text { México } & \text { Otzoloapan } & 24.8 \\ \text { México } & \text { Tlatlaya } & 26.4 \\ \text { México } & \text { Zacualpan } & 28.2 \\ \text { México } & \text { Villa de Allende } & 28.5 \\ \text { México } & \text { Sultepec } & 30 \\ \text { México } & \text { Ixtapan del Oro } & 31.8 \\ \text { México } & \text { Luvianos } & 32.1 \\ \text { México } & \text { Donato Guerra } & 33.6\end{array}$

Tabla 2. Datos originales. Fuente; Elaboración propia con base en CONEVAL (2015). 